\title{
Reconstrução de um Defeito Cirúrgico Grande da Glabela
}

\author{
Alexandre Miroux Catarino', Maria Goreti Catorze², José Manuel Labareda², Isabel Viana ${ }^{3}$ \\ 'Médico Interno de Dermatovenereologia/Resident of Dermatology and Venereology, Serviço de Dermatologia, Centro Hospitalar \\ de Lisboa Ocidental, Lisboa, Portugal \\ ${ }^{2}$ Assistente Hospitalar Graduada de Dermatovenereologia, Serviço de Dermatologia, Centro Hospitalar de Lisboa Ocidental, \\ Lisboa, Portugal / Consultant of Dermatology and Venereology, Dermatology Department, Centro Hospitalar de Lisboa Ocidental, \\ Lisbon, Portugal \\ ${ }^{3}$ Chefe de Serviço de Dermatovenereologia, Serviço de Dermatologia, Centro Hospitalar de Lisboa Ocidental, Lisboa, Portugal / \\ Department Director of Dermatology and Venereology, Dermatology Department, Centro Hospitalar de Lisboa Ocidental, Lisbon, \\ Portugal
}

RESUMO - A reconstrução de defeitos grandes da glabela, sobretudo quando envolvem a fronte, é um desafio cirúrgico. A reconstrução cirúrgica desta localização deve ter em consideração as subunidades estéticas, evitar a aproximação dos supracílios e manter a simetria da face. É apresentado um caso de reconstrução de defeito cirúrgico envolvendo a glabela e a fronte pós cirurgia de Mohs. Optou-se pela realização de retalho de avanço A-T para encerramento da porção superior do defeito, associado a retalho de transposição romboidal para encerramento da zona inferior de forma a manter o espaçamento entre as supracílios. A reconstrução por subunidades estéticas e a associação de retalhos permitiu um bom resultado estético e funcional.

PALAVRAS-CHAVE - Carcinoma Basocelular; Neoplasias da Pele/cirurgia; Procedimentos Cirúrgicos Reconstrutivos; Retalhos CirúrgicosTesta/cirurgia.

\section{Reconstruction of a Large Defect of the Glabella}

ABSTRACT - The reconstruction of large defects of the glabella, especially when involving the forehead, is a surgical challenge. Surgical reconstruction of this location should take into account aesthetic subunits, avoid approximation of eyebrows and maintain symmetry of the face.

The authors present a case with reconstruction of a surgical defect involving the glabella and the forehead after Mohs surgery. An A-T advancement flap to close the superior portion of the defect was combined with a rhomboidal transposition flap for closing the lower zone in order to maintain the distance between the eyebrows. Reconstruction by aesthetic subunits and the association of flaps allowed a good aesthetic and functional result.

KEYWORDS - Forehead/surgery; Neoplasms, Basal Cell; Reconstructive Surgical Procedures; Skin Neoplasms/surgery; Surgical Flaps.

\section{INTRODUÇÃO}

A glabela é a subunidade estética delimitada pela fronte superiormente, os supracílios lateralmente e a raiz nasal inferiormente. ' A reconstrução de defeitos cirúrgicos de grandes dimensões envolvendo a glabela e a fronte corresponde a um desafio cirúrgico, quer pelas estruturas contíguas existentes quer pela importância de manter o espaço interciliar e simetria dos supracílios.

\section{CASO CLÍNICO}

Doente do sexo feminino de 51 anos com história de carcinoma basocelular (CBC) submetido a uma intervenção cirúrgica e crioterapia prévias nos últimos 3 anos. Esteve sem seguimento durante o último ano, tendo sido enviada à consula de Dermatologia do Hospital Egas Moniz por suspeita de recidiva de $C B C$ para ser submetida a cirurgia micrográfica de Mohs. Ao exame objetivo verificava-se presença de placa
Correspondência: Alexandre Miroux Catarino Serviço de Dermatologia, Hospital Egas Moniz Rua da Junqueira 126

1349-019 Lisboa, Portugal

E-mail: alexandre_catarino@hotmail.com

DOI: https://dx.doi.org/10.29021/spdv.77.1.1009
Recebido/Received

12 Novembro/November 2018

Aceite/Accepted

29 Dezembro/December 2018 


\section{Pérolas Cirúrgicas}

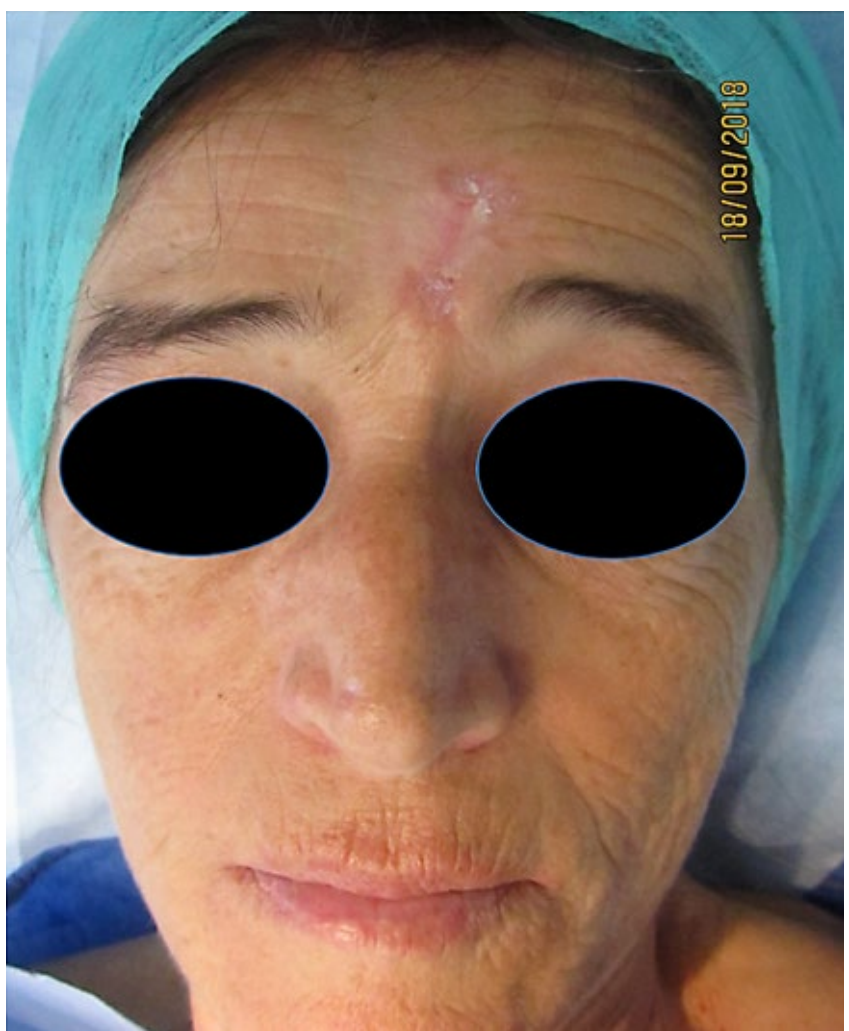

Figura 1 - Lesão envolvendo a glabela e fronte.

eritematosa de centro deprimido com aspeto cicatricial, limites mal definidos, bordo bosselado e áreas erosionadas. A lesão media 1,9 × 3,4 cm de diâmetro e localizava-se na glabela e área contígua da fronte (Fig. 1).

Além desta lesão verificava-se na metade direita da ponta nasal pápula da cor da pele envolvente, deprimida, com telangiectasias arboriformes e bordo perolado, sugestiva de carcinoma basocelular (Fig. 2). Os dois tumores foram abordados no mesmo tempo cirúrgico.

Como antecedentes pessoais destaca-se história de melanoma maligno da coxa (fino, nível II de Clark) excisado há 5 anos.

A doente foi submetida a cirurgia micrográfica de Mohs sob anestesia local com lidocaína a 2\% e bupivacaína a $0,25 \%$ sem adrenalina. Após exérese da lesão clinicamente aparente da glabela, removeu-se o tecido correspondendo à margem lateral e profunda para controlo pelo método de Mohs a fresco (Fig. 3). As margens foram negativas no primeiro estadio de Mohs, tendo resultado um defeito grosseiramente ovalado de grandes dimensões $(4 \times 2,5 \mathrm{~cm}$ de maiores eixos) envolvendo a glabela e a fronte (Fig. 4).

Nesta fase foram avaliadas as possíveis opções reconstrutivas. Pelo resultado cosmético pouco aceitável, descartou-se a possibilidade de cicatrização por segunda intenção, o encerramento direto e a colocação de enxerto de pele total.

Tendo em vista o melhor resultado cosmético e funcional dividiu-se o defeito por subunidades estéticas para orientar a

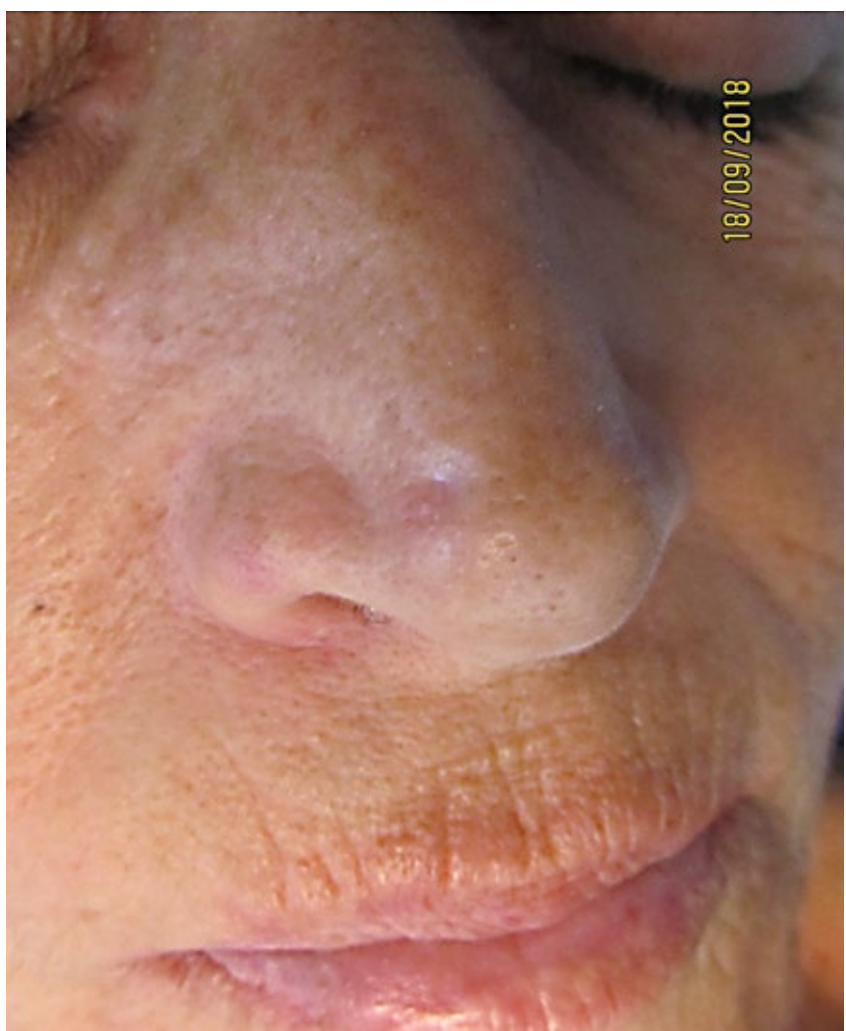

Figura 2 - Pápula deprimida na ponta nasal correspondendo a CBC.

reconstrução. A subunidade correspondendo à porção superior do defeito (zona 1), localizada acima da linha que une o limite superior dos supracílios e a subunidade inferior correspondendo à glabela (zona 2) (Fig. 5).

Para reconstrução do defeito da fronte optou-se pelo retalho de avanço bilateral A-T, com incisão ao longo do bordo superior dos supracílios (Fig.s 5 e 6). Para reconstrução da

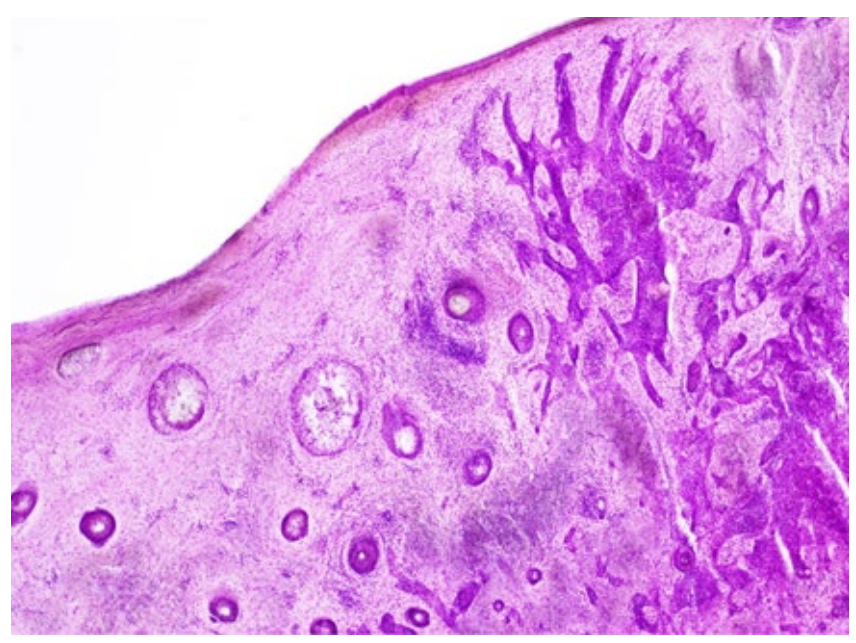

Figura 3 - Presença de carcinoma basocelular na peça de excisão da lesão clinicamente aparente em cortes a fresco. 


\section{Pérolas Cirúrgicas}

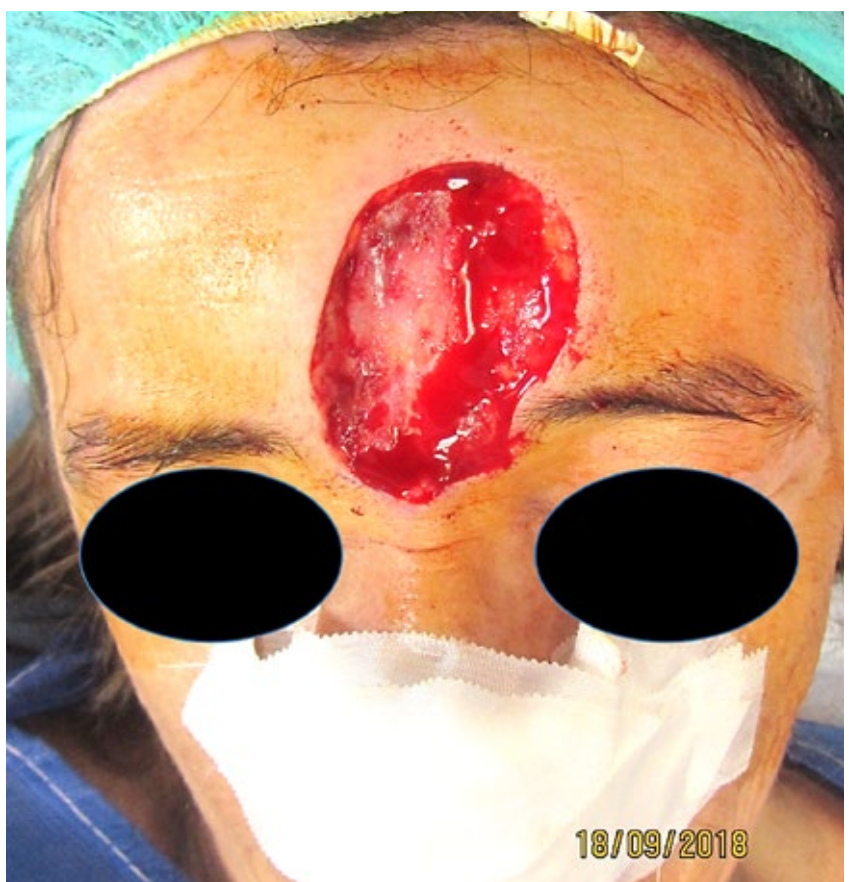

Figura 4 - Defeito cirúrgico da glabela e fronte.

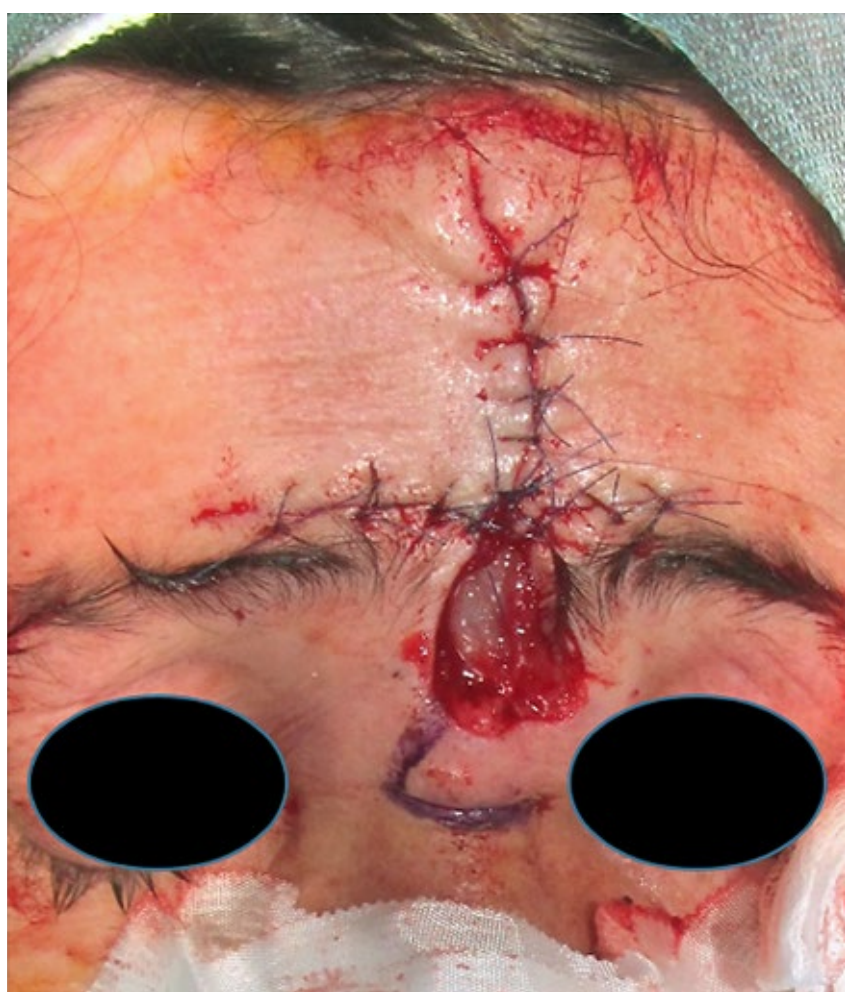

Figura 6 - Defeito remanescente após realização do retalho de avanço A-T. Desenho do retalho de transposição romboidal a realizar para encerramento do defeito da glabela.

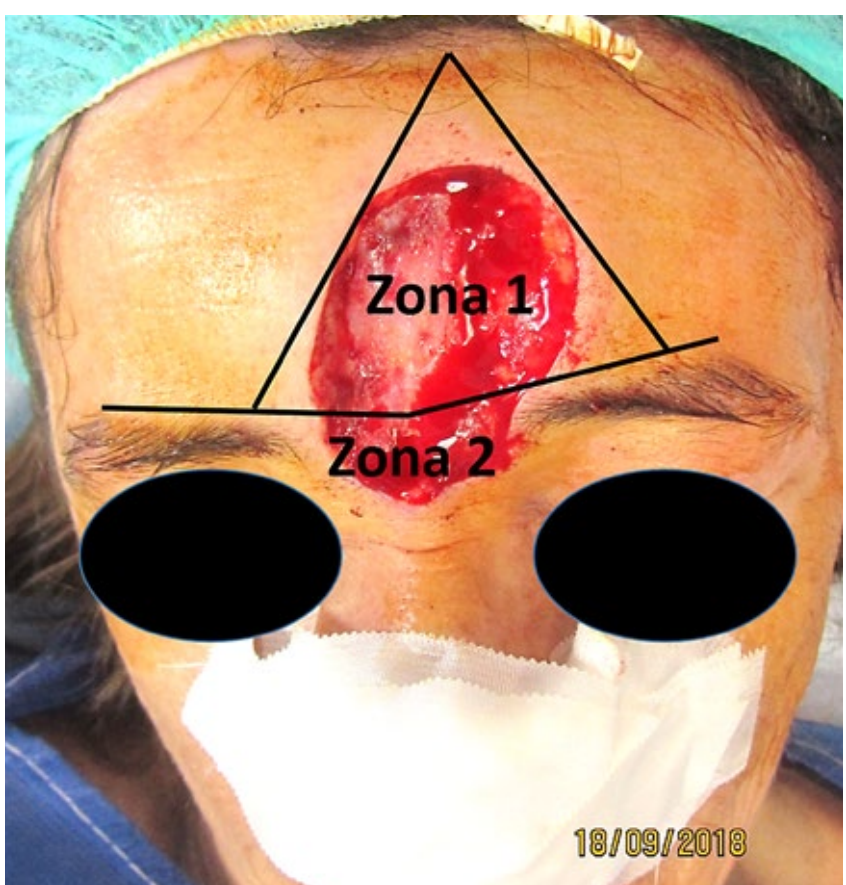

Figura 5 - Divisão do defeito em 2 subunidades - zona 1 e zona 2 - e desenho do retalho de avanço A-T.

região inferior do defeito optou-se por um retalho de transposição romboidal, de forma a evitar a união dos supracílios e, simultaneamente, tensão sobre o canto interno do olho (Fig. 6).

A lesão da ponta nasal foi excisada no mesmo tempo operatório com controlo de margens pelo método de Mohs a fresco e realizado o encerramento do defeito com retalho de rotação (Fig.s 7 e 8).

No pós-operatório imediato verificou-se uma ligeira elevação dos supracílios, mas a manutenção da simetria e espaço entre eles (Fig. 9). Às 7 semanas o resultado cosmético e funcional era bom, estando a doente satisfeita (Fig. 10).

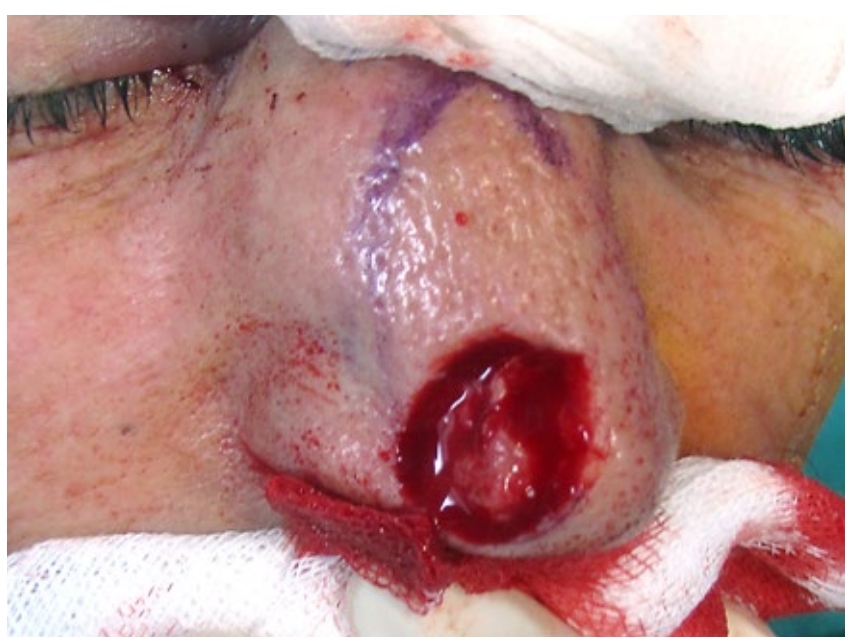

Figura 7 - Defeito cirúrgico da ponta nasal. 


\section{Pérolas Cirúrgicas}

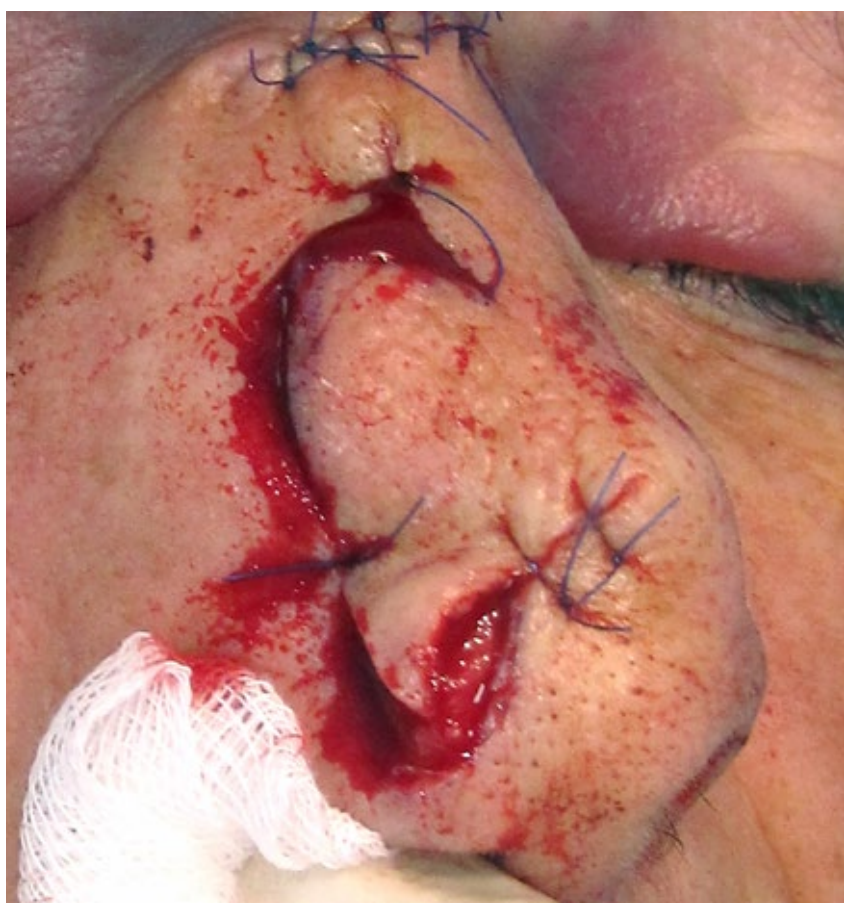

Figura 8 - Passo intermédio na realização do retalho de rotação para reconstrução do defeito da ponta nasal.

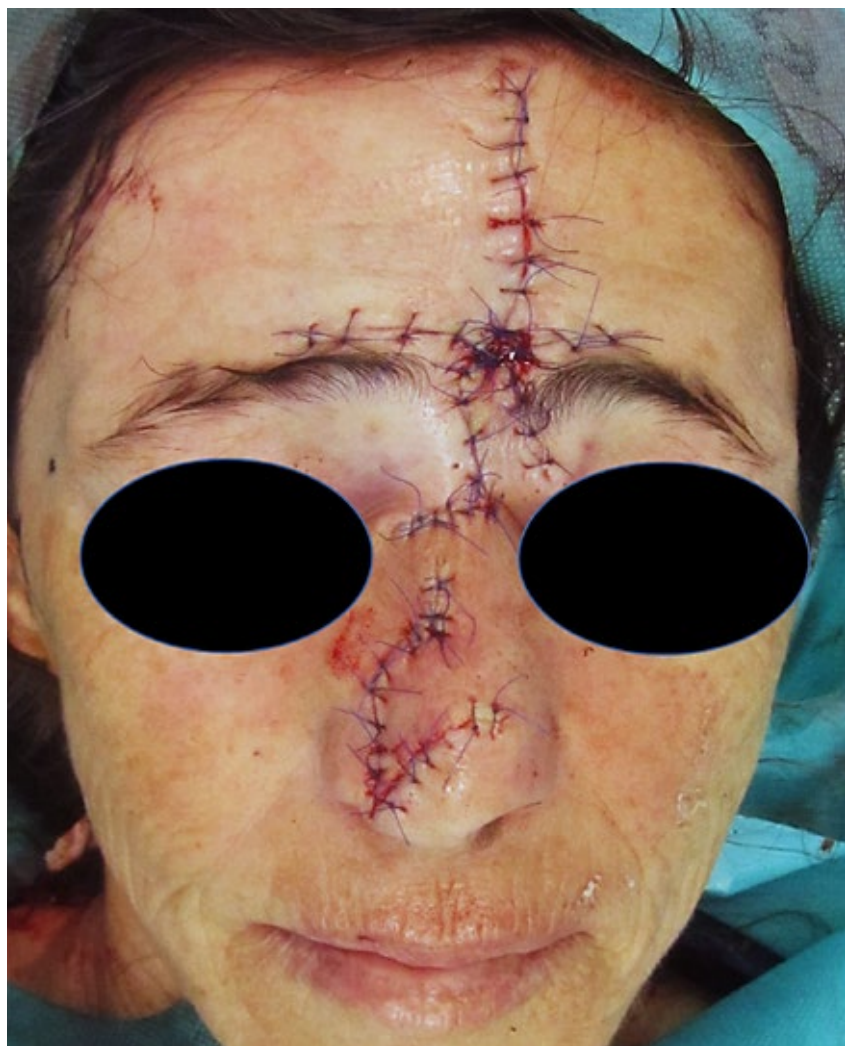

Figura 9 - Pós-operatório imediato. Reconstrução do defeito da glabela e fronte e do defeito pós exérese de CBC da ponta nasal com retalho de rotação.

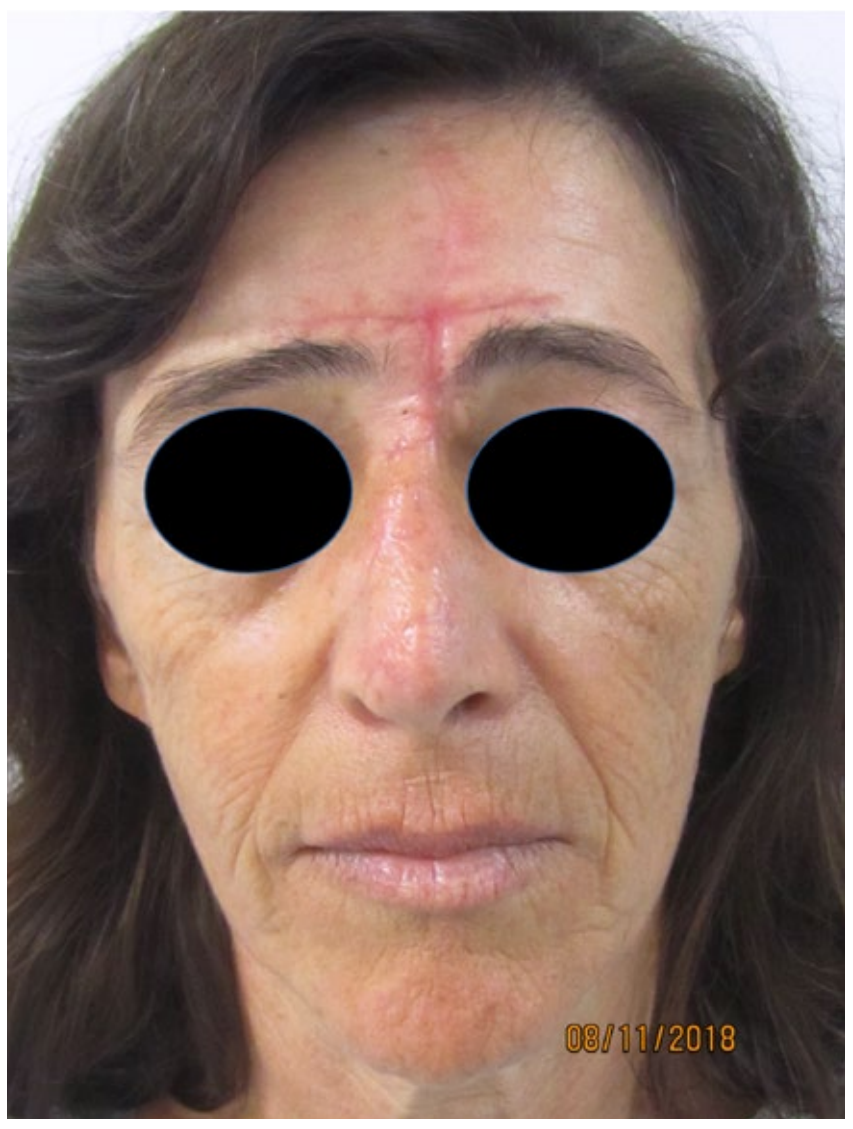

Figura 10 - Resultado às 7 semanas do pós-operatório.

\section{DISCUSSÃO}

A reconstrução de grandes defeitos cirúrgicos envolvendo a glabela e a fronte é um desafio cirúrgico. A cicatrização por segunda intenção é demorada, associa-se a um resultado cosmético pouco previsível e a retração da cicatriz pode provocar desvio do canto interno do olho e distorção dos supracílios. ${ }^{2}$ Defeitos grandes inviabilizam o encerramento direto pelo inaceitável resultado cosmético e funcional. A colocação de enxerto associa-se a diferenças de textura e cor da pele além da possível retração aquando da cicatrização.

Em defeitos que atingem diferentes unidades cosméticas a utilização de múltiplos retalhos em simultâneo é uma arma útil na reconstrução. ${ }^{3}$

Na reconstrução de defeitos centrais da fronte, o retalho de avanço A-T é uma das técnicas de escolha habitual. Este retalho pode ser utilizado em defeitos grandes, permite esconder as suturas horizontais nas rugas e a sutura vertical tem pouco impacto cosmético. Aquando de defeitos contíguos ou envolvendo parte da glabela, é importante que a incisão acompanhe a curvatura dos supracílios, o que permite uma melhor distribuição da tensão e uma redução mínima da distância entre elas. ${ }^{4}$

Dado o envolvimento da totalidade da glabela até à raiz do nariz, não foi possível encerrar o defeito com o retalho de avanço A-T da fronte. $O$ encerramento direto não é uma 


\section{Pérolas Cirúrgicas}

opção em defeitos importantes da glabela, sendo essencial trazer pele de outra localização de forma a não reduzir a distância entre os supracílios. O retalho de transposição romboidal utilizado é um retalho simples e que não origina tensão nas estruturas envolventes, nomeadamente no canto interno do olho.

Da revisão da literatura realizada, verificou-se que Blake e Maher descreveram uma caso com um defeito cirúrgico semelhante e mesma opção reconstrutiva. ${ }^{2}$

Conflitos de interesse: Os autores declaram não possuir conflitos de interesse.

Suporte financeiro: $O$ presente trabalho não foi suportado por nenhum subsídio ou bolsa.

Confidencialidade dos dados: Os autores declaram ter seguido os protocolos do seu centro de trabalho acerca da publicação dos dados de doentes.

Direito a privacidade e consentimento escrito: Os autores declaram que pediram consentimento para usar as imagens no artigo.

Consentimento do Doente: Obtido.

Conflicts of interest: The authors have no conflicts of interest to declare.

Financing Support: This work has not received any contribution, grant or scholarship.

Confidentiality of data: The authors declare that they have followed the protocols of their work center on the publication of data from patients.

Privacy policy and informed consent: The authors declare that have the written informed consent for the use of patient's photos in this article.

Patient consent: Obtained.
Proveniência e revisão por pares: Não comissionado; revisão externa por pares

Provenance and peer review: Not commissioned; externally peer reviewed

\section{REFERÊNCIAS}

1. Martinez SA, Masa I, Salinas Velasco V. Nasal root island flap for reconstruction of glabellar defects. Ann Plast Surg. 2015;74:34-6. doi: 10.1097/ SAP.0b013e31828d9969.

2. Blake B, Maher I. Reconstruction of a large defect of the glabella and forehead. Dermatol Surg. 2015;41:2802. doi: 10.1097/DSS.0000000000000205.

3. Goulão J, Alves J, António AM. Reconstrução de um defeito cirúrgico complexo do canto interno. Rev Soc Port Dermatol Venereol; 2018;76:201-4. doi. org/10.29021/spdv.76.2.915.

4. Puviani M, Curci M. The "Batman flap": a novel technique to repair a large central glabellar defect. Int J Dermatol. 2018;57:477-9. doi: 10.1111/ijd.13844. 